\title{
A Method against Interrupted-Sampling Repeater Jamming Based on Energy Function Detection and Band-Pass Filtering
}

\author{
Hui Yuan, Chun-yang Wang, Xin Li, and Lei An \\ Air and Missile Defense College of Air Force Engineering University, Xian, Shanxi 710051, China \\ Correspondence should be addressed to Chun-yang Wang; wcy_kgd_cn@163.com
}

Received 24 November 2016; Revised 26 February 2017; Accepted 12 March 2017; Published 30 March 2017

Academic Editor: Atsushi Mase

Copyright (C) 2017 Hui Yuan et al. This is an open access article distributed under the Creative Commons Attribution License, which permits unrestricted use, distribution, and reproduction in any medium, provided the original work is properly cited.

\begin{abstract}
Interrupted-sampling repeater jamming (ISRJ) is a new kind of coherent jamming to the large time-bandwidth linear frequency modulation (LFM) signal. Many jamming modes, such as lifelike multiple false targets and dense false targets, can be made through setting up different parameters. According to the "storage-repeater-storage-repeater" characteristics of the ISRJ and the differences in the time-frequency-energy domain between the ISRJ signal and the target echo signal, one new method based on the energy function detection and band-pass filtering is proposed to suppress the ISRJ. The methods mainly consist of two parts: extracting the signal segments without ISRJ and constructing band-pass filtering function with low sidelobe. The simulation results show that the method is effective in the ISRJ with different parameters.
\end{abstract}

\section{Introduction}

Interrupted-sampling repeater jamming (ISRJ) [1] based on the digital radio frequency memory (DRFM) technology is a new kind of smart jamming, which aims especially at the linear frequency modulation (LFM) signals with large timebandwidth product. The prominent characteristics of ISRJ are embodied in its antenna system and its manner of sampling and storing signals. ISRJ can be used in receive-transmit time-sharing antenna system as well as antenna system with two receive-transmit antennas which work asynchronously. Besides, sampling and storing the whole radar signal without distortion are not necessary. Therefore, the problems of highspeed sampling and receive-transmit antenna isolation are settled satisfactorily, which means great value of practical application on the occasions of small jammer-platform like missile-borne jammer.

Compared with ISRJ, the Chopping and Interleaving (C\&I) proposed in [2] by Sparrow and Cakilo can only be accomplished in the next or after several radar pulse repetition periods and the signal is continuous, while the ISRJ signal can be generated in the current radar pulse repetition period and the signal is intermittent, which leads the methods such as phase perturbation [3], adjusting the chirp rate [4], and pulse diversity [5-8] to failure. The fundamental ISRJ, whose repeat sampling interval is constant and the duty ratio is $50 \%$, has many deficiencies. For example, the amplitudes of the subfalse targets decay rapidly and the main false target is always behind the true target on the self-defense occasion. For solving these problems, some further studies have been presented like interrupted-sampling and periodic repeater jamming [9], irregularly interrupted-sampling and repeater jamming [10], interrupted-sampling and nonuniform periodic repeater jamming [11], and interrupted-sampling and successive repeater jamming [12]. For sharing the common operating mode "storage-repeater-storage-repeater," they are collectively called ISRJ in this paper. Compared with the ISRJ with fixed repeat sampling interval, interrupted-sampling and periodic repeater jamming and interrupted-sampling and successive repeater jamming can form a mass of lifelike false targets and have obvious advantages in fidelity and amount of false targets, flexibility of system, computation burden, and requirement of hardware environment [13]. The ISRJ with irregular sampling periods and interruptedsampling and nonuniform periodic repeater jamming can achieve the desired jamming effect through setting up different parameters like sampling pulse width, delay time of repeater, pulse width of repeater, and repeat sampling 
interval. Moreover, the false targets with different amplitudes can be generated without changing the instantaneous transmit power which reduce the complexity of hardware system and the energy loss caused by the power switching frequently. Besides the studies on jamming methods, Feng made some researches on the performance of ISRJ on monopulse radar [14], dechirping radar [15], and the impact of ISJR on radar constant false alarm rate (CFAR) detection [16]. There are also many researches on 2-dimension (2D) ISRJ methods and their application on synthetic aperture radar (SAR) or inverse synthetic aperture radar (ISAR) [17-19].

Due to the sensitivity of the military, there is only limited public research literature on electronic countercountermeasure (ECCM) schemes with respect to the extensive researches on ISRJ. As a coherent repeater jamming method, ISRJ has both deception and blanket jamming effects under certain parameters and cannot be suppressed by traditional radar antijamming methods such as matched filter and coherent integration. Furthermore, ISRJ is mostly used as self-defense jamming, so the traditional sidelobe cancellation and sidelobe blanking are also useless. Aiming at the recognition of ISRJ, Jiang et al. [20] have put forward some recognition methods by using the differences on frequency spectrum of the jamming signal and the target signal, which is really important for further research on ECCM methods against ISRJ. According to the discontinuity in time domain of the ISRJ signal, Gong et al. [21] have proposed an effective ECCM scheme for eliminating the ISRJ-based false targets from the pulse compression result based on the timefrequency analysis method. The segments not disturbed by ISRJ are detected by finding the modulus minimum of the short-time Fourier transform (STFT) result, and the bandpass filter is constructed to filter out the ISRJ signal. However, Gong does not give a method to determine the length of the time unit in which only the target echo signal exists.

In this paper, a new method against the ISRJ is proposed based on the "storage-repeater-storage-repeater" characteristics of the ISRJ and the differences in the time-frequencyenergy domain between the ISRJ signal and the target signal. The new method does not need to make the time-frequency analysis such as short-time Fourier transform and only needs to compute the simple energy function of the radar signal. Then the signal segments not disturbed by the ISRJ are obtained by comparing the energy function with a threshold value. The rest of the paper is organized as follows. Section 2 first reviews the signal models of the target echo signal and the ISRJ signal before and after the dechirping processing, respectively, and then analyzes their time-frequency-energy domain characteristics. Section 3 puts forward the antijamming method based on the energy function detection and band-pass filtering. The methods of extracting the signal segments without ISRJ, determining the threshold value, and building the band-pass filtering function with low sidelobes are presented. Section 4 carries out some simulations to demonstrate that the proposed ECCM method is effective in ISRJ with different parameters resulting in different jamming results including the multiple lifelike false targets and the intensively distributed false targets.

\section{Signal Model and Properties on Time-Frequency-Energy Domain}

2.1. Signal Model. Dechirping processing is one of the common methods of wideband LFM signal, which can convert the time delay of the target echo signal into frequency; that is, the target echo signal after dechirping is a singlefrequency signal whose frequency is proportional to the target distance. Then, the pulse compression result can be obtained by doing the Fourier transform to the radar echo signal after dechirping processing. This method can not only keep the high range-resolution but also reduce the radar's intermediate frequency (IF) bandwidth. ISRJ can also form effective multiple false targets to the dechirping wideband LFM pulse compression radar [16]. Furthermore, the prime component of the jamming signal's pulse compression output can be ahead of the target by controlling the time delay of repeater, which means that the spatial distributions of the false targets are more flexible.

Assume that the normalized LFM signal emitted by the radar is

$$
s(t)=\operatorname{rect}\left(\frac{t-T / 2}{T}\right) \exp \left[j 2 \pi\left(f_{c} t+\frac{1}{2} k t^{2}\right)\right],
$$

where

$$
\operatorname{rect}\left(\frac{t}{T}\right)= \begin{cases}1, & -\frac{T}{2} \leq t \leq \frac{T}{2} \\ 0, & \text { else }\end{cases}
$$

represents a rectangular pulse with pulse width $T$ and center location $0, f_{c}$ is the radar carrier frequency, $k=B / T$ represents the chirp rate, and $B$ is the bandwidth of the signal. For the convenience of researching, assume that the true target used in this paper just has one scatter point and the distance between them is $R_{t}$. Then, the target echo signal can be denoted as

$$
\begin{aligned}
s_{t}(t)= & A_{r} \operatorname{rect}\left(\frac{t-T / 2-\tau_{t}}{T}\right) \\
& \times \exp \left\{j 2 \pi\left[f_{c}\left(t-\tau_{t}\right)+\frac{1}{2} k\left(t-\tau_{t}\right)^{2}\right]\right\},
\end{aligned}
$$

where $\tau_{t}=2 R_{t} / c$ is the time delay of the target echo signal, $c$ is the velocity of light, and $A_{r}=\sqrt{2 P_{t} G^{2} \lambda^{2} \sigma /(4 \pi)^{3} R_{t}^{4}}$ is the amplitude of the target echo signal. $P_{t}, G, \lambda, \sigma$ are the peak power of the radar signal, antenna gain, wavelength, and the target radar cross section (RCS), respectively.

The reference signal of dechirping radar can be expressed by

$$
\begin{aligned}
s_{\text {ref }}(t)= & \operatorname{rect}\left(\frac{t-T_{\text {ref }} / 2-\tau_{\text {ref }}}{T_{\text {ref }}}\right) \\
& \times \exp \left\{j 2 \pi\left[f_{c}\left(t-\tau_{\text {ref }}\right)+\frac{1}{2} k\left(t-\tau_{\text {ref }}\right)^{2}\right]\right\},
\end{aligned}
$$

where $\tau_{\text {ref }}=2 R_{\text {ref }} / c, R_{\text {ref }}$ is the reference range, $T_{\text {ref }}$ is the receiving window, and generally $T_{\text {ref }}>T$. 
The dechirping processing consists of mixing radar signals and reference signal and low-pass filtering. Using the analytic signal, the target echo signal after dechirping can be denoted as

$$
\begin{aligned}
s_{t \_\mathrm{de}} & =s_{t}(t) \cdot s_{\mathrm{ref}}^{*}(t)=A_{r} \operatorname{rect}\left(\frac{t-T / 2-\tau_{t}}{T}\right) \\
& \times \exp \left[j 2 \pi \left(-k\left(\tau_{t}-\tau_{\mathrm{ref}}\right) t-f_{c}\left(\tau_{t}-\tau_{\mathrm{ref}}\right)\right.\right. \\
& \left.\left.+\frac{1}{2} k\left(\tau_{t}^{2}-\tau_{\mathrm{ref}}^{2}\right)\right)\right] .
\end{aligned}
$$

$$
s_{j}(t)=A_{j} \sum_{m=1}^{M}\left(\sum_{n=0}^{N-1} \operatorname{rect}\left(\frac{t-\tau / 2-\tau_{t}-\tau_{d}-m \tau-n T_{s}}{\tau}\right) \times \exp \left[j 2 \pi\left(f_{c}\left(t-m \tau-\tau_{t}-\tau_{d}\right)+\frac{1}{2} k\left(t-m \tau-\tau_{t}-\tau_{d}\right)^{2}\right)\right]\right),
$$

The basic idea of ISRJ is "storage-repeater-storagerepeater," which means that the jammer firstly samples and stores parts of the radar signal and then transmits it once or more than once, uniformly or nonuniformly to the radar. Repeat like this until the end of the radar signal. Without loss of generality, we analyze the interrupted-sampling and periodic repeater jamming. Figure 1 shows the jamming principle. $T_{s}$ is the repeat sampling interval. $\tau$ is the sampling pulse width. $T_{s}$ is $M+1$ times of $\tau$. Then the interruptedsampling and periodic repeater jamming can be described by where $\tau_{d}$ denotes the delay of the jammer and $A_{j}=$ $\sqrt{2 P_{j} G \lambda^{2} /\left(4 \pi R_{t}\right)^{2}}$ denotes the amplitude of the jamming signal. $P_{j}$ is the transmit power of the jammer. Then the jamming signal after dechirping can be expressed by

$$
\begin{aligned}
& s_{j_{-} \mathrm{de}}=s_{j}(t) \cdot s_{\mathrm{ref}}^{*}(t)=A_{j} \sum_{m=1}^{M}\left(\sum_{n=0}^{N-1} \operatorname{rect}\left(\frac{t-\tau / 2-\tau_{t}-\tau_{d}-m \tau-n T_{s}}{\tau}\right)\right. \\
& \left.\quad \cdot \exp \left\{j 2 \pi\left[-k\left(m \tau+\tau_{t}+\tau_{d}-\tau_{\mathrm{ref}}\right) t-f_{c}\left(m \tau+\tau_{t}+\tau_{d}-\tau_{\mathrm{ref}}\right)+\frac{1}{2} k\left(m \tau+\tau_{t}+\tau_{d}\right)^{2}-\frac{1}{2} k \tau_{\mathrm{ref}}^{2}\right]\right\}\right) .
\end{aligned}
$$

2.2. Properties on Time-Frequency-Energy Domain. The instantaneous frequency of a signal is defined by [22] as the derivative of phase with respect to time.

$$
f=\frac{1}{2 \pi} \frac{d \varphi(t)}{d t} .
$$

Then according to (3), (5), (6), and (7), the instantaneous frequency of the target echo signal and the jamming signal before and after dechirping processing can be obtained as follows:

$$
\begin{aligned}
f_{t}= & \frac{1}{2 \pi} \operatorname{rect}\left(\frac{t-T / 2-\tau_{t}}{T}\right) \cdot \frac{d}{d t}\left[f_{c}\left(t-\tau_{t}\right)+\frac{1}{2} k\left(t-\tau_{t}\right)^{2}\right]=\operatorname{rect}\left(\frac{t-T / 2-\tau_{t}}{T}\right)\left[f_{c}+k\left(t-\tau_{t}\right)\right], \\
f_{t_{-} \mathrm{de}} & =\frac{1}{2 \pi} \operatorname{rect}\left(\frac{t-T / 2-\tau_{t}}{T}\right) \cdot \frac{d}{d t}\left\{2 \pi\left[-k\left(\tau_{t}-\tau_{\mathrm{ref}}\right) t-f_{c}\left(\tau_{t}-\tau_{\mathrm{ref}}\right)+\frac{1}{2} k\left(\tau_{t}^{2}-\tau_{\mathrm{ref}}^{2}\right)\right]\right\}=\operatorname{rect}\left(\frac{t-T / 2-\tau_{t}}{T}\right) \\
& \cdot\left(-k \Delta \tau_{t}\right), \Delta \tau_{t}=\tau_{t}-\tau_{\mathrm{ref}}, \\
f_{j}= & \frac{1}{2 \pi} \sum_{m=1}^{M} \sum_{n=0}^{N-1} \operatorname{rect}\left(\frac{t-\tau / 2-\tau_{t}-\tau_{d}-m \tau-n T_{s}}{\tau}\right) \cdot \frac{d}{d t}\left[2 \pi\left[f_{c}\left(t-m \tau-\tau_{t}-\tau_{d}\right)+\frac{1}{2} k\left(t-m \tau-\tau_{t}-\tau_{d}\right)^{2}\right]\right] \\
& =\sum_{m=1}^{M} \sum_{n=0}^{N-1} \operatorname{rect}\left(\frac{t-\tau / 2-\tau_{t}-\tau_{d}-m \tau-n T_{s}}{\tau}\right) \cdot\left[f_{c}+k\left(t-m \tau-\tau_{t}-\tau_{d}\right)\right],
\end{aligned}
$$




$$
\begin{aligned}
f_{j_{-} \mathrm{de}} & =\frac{1}{2 \pi} \sum_{m=1}^{M} \sum_{n=0}^{N-1} \operatorname{rect}\left(\frac{t-\tau / 2-\tau_{t}-\tau_{d}-m \tau-n T_{s}}{\tau}\right) \\
& \frac{d}{d t}\left\{2 \pi\left[-k\left(m \tau+\tau_{t}+\tau_{d}-\tau_{\text {ref }}\right) t-f_{c}\left(m \tau+\tau_{t}+\tau_{d}-\tau_{\text {ref }}\right)+\frac{1}{2} k\left(m \tau+\tau_{t}+\tau_{d}\right)^{2}-\frac{1}{2} k \tau_{\text {ref }}^{2}\right]\right\} \\
= & \sum_{m=1}^{M} \sum_{n=0}^{N-1} \operatorname{rect}\left(\frac{t-\tau / 2-\tau_{t}-\tau_{d}-m \tau-n T_{s}}{\tau}\right)\left(-k \Delta \tau_{j}\right), \quad \Delta \tau_{j}=m \tau+\tau_{t}+\tau_{d}-\tau_{\text {ref }} .
\end{aligned}
$$

According to (9), we can realize the TF distribution of the target signal and the jamming signal before and after dechirping by Figure 2. It can be seen from Figure 2 that the TF distribution of the jamming signal is discontinuous no matter before or after dechirping because the ISRJ jammer does not transmit the jamming signal when it is receiving the radar signal, which is also the main advantage of ISRJ. However, in pulse duration, the target echo signal before and after dechirping is continuously changing with time. Especially after dechirping, the frequency of the target echo signal is invariable. Based on the great difference between the TF characteristics of the ISRJ signal and the target echo signal, we can build a band-pass filter using the target echo signal segments which are not disturbed by the ISRJ signal to eliminate most of the false targets.

Using the energy function defined as the square of the signal's modulus, we analyze the distribution characteristics of the radar signal in the time-energy domain under the jamming condition. The energy functions of the target echo signal $s_{t_{-} \text {de }}(t)$, the ISRJ signal $s_{j_{-} \text {de }}(t)$, and the radar received signals $x(t)=s_{t \_d e}(t)+s_{j_{-} \text {de }}(t)$ under the jamming condition after dechirping are

$$
\begin{aligned}
& E_{t}(t)=\left|s_{t_{-} \mathrm{de}}(t)\right|^{2}=A_{r}^{2} \operatorname{rect}\left(\frac{t-T / 2-\tau_{t}}{T}\right), \\
& E_{j}(t)=\left|s_{j_{-} \text {strech }}(t)\right|^{2} \\
& =A_{j}^{2} \sum_{n=0}^{N-1} \operatorname{rect}\left(\frac{t-\tau_{t}-\tau_{d}-(1+M / 2) \tau-n T_{s}}{M \tau}\right), \\
& E_{x}(t)=\left|s_{r_{-} \text {strech }}(t)+s_{j_{-} \text {strech }}(t)\right|^{2} \\
& \quad=A_{r}^{2} \operatorname{rect}\left(\frac{t-T / 2-\tau_{t}}{T}\right) \\
& \quad+A_{j}^{2} \sum_{n=0}^{N-1} \operatorname{rect}\left(\frac{t-\tau_{t}-\tau_{d}-(1+M / 2) \tau-n T_{s}}{M \tau}\right) \\
& \quad+2 A_{r} A_{j} \sum_{m=1}^{M} \sum_{n=0}^{N-1} \operatorname{rect}\left(\frac{t-\tau_{t}-\tau_{d}-\tau / 2-m \tau-n T_{s}}{\tau}\right) \\
& \quad \times \cos \left[2 \pi k\left(m \tau+\tau_{d}\right) t+\varphi\right],
\end{aligned}
$$

respectively, where $\varphi=2 \pi\left[f_{c}\left(m \tau+\tau_{d}\right)+(1 / 2) k \tau_{t}^{2}-\right.$ $\left.(1 / 2) k\left(m \tau+\tau_{t}+\tau_{d}\right)^{2}\right]$.
For $\left|\cos \left[2 \pi k\left(m \tau+\tau_{d}\right) t+\varphi\right]\right| \leq 1, E_{x}(t)$ satisfies $\left(A_{j}-\right.$ $\left.A_{r}\right)^{2} \leq E_{x}(t) \leq\left(A_{j}+A_{r}\right)^{2}$ during transmitting the jamming signals. Figure 3 shows the curves of the energy functions of the target echo signal, the ISRJ signal, and the radar received signals under the jamming condition after dechirping.

It can be seen from Figure 3 that, during the sampling duration, the energy is stable and small because the jammer does not transmit the jamming signal and only the target echo signal is received, while the energy is fluctuant quickly and strong during the time of transmitting the jamming signal.

\section{ECCM Scheme against ISRJ}

3.1. Executing Steps of ECCM Scheme. According to the above analysis, an ECCM scheme against the ISRJ can be summarized by the following steps.

Step 1. Calculate energy function of $x(t)$ (see (12)).

Step 2. Determine a threshold value $\gamma$.

Step 3. Extract the signal segments not disturbed by the ISRJ signal.

For any time $t_{0}$ during the radar received signals, when $E_{x}\left(t_{0}\right)<\gamma, p\left(t_{0}\right)=1$; otherwise, $p\left(t_{0}\right)=0$. Then the signal segments not disturbed by ISRJ signal can be expressed as

$$
h(t)=x(t) \cdot p(t) .
$$

Step 4. Get the band-pass filtering function $H(f)$ by doing the Fourier transform on $h(t)$ and then carry out the normalization process.

$$
H(f)=\frac{\int_{t} h(t) \cdot e^{-j 2 \pi f t} d t}{\max \left(\left|\int_{t} h(t) \cdot e^{-j 2 \pi f t} d t\right|\right)} .
$$

Doing the Fourier transform on $x(t)$, we can get the pulse compression result with ISRJ signal $X(f)$.

$$
X(f)=\int_{t} x(t) \cdot e^{-j 2 \pi f t} d t .
$$

Step 5 (filtering). Multiplying $X(f)$ with the band-pass filtering function $H(f)$, the ISRJ can be suppressed and then the final pulse compression result can be obtained as

$$
Y(f)=H(f) \cdot X(f) .
$$




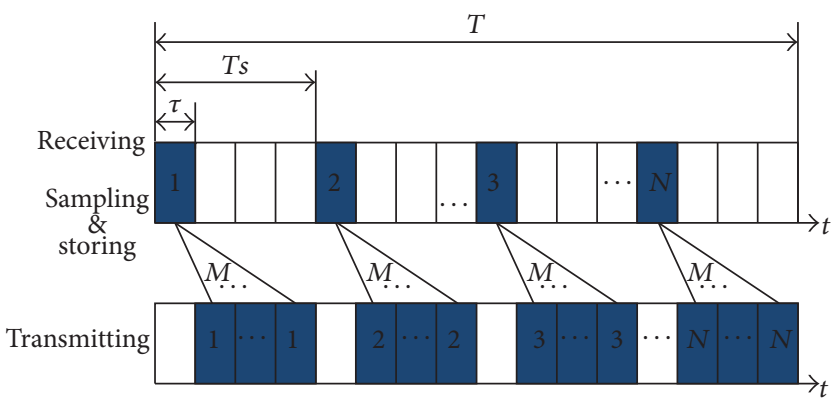

FIGURE 1: The principle of the interrupted-sampling and periodic repeater jamming.

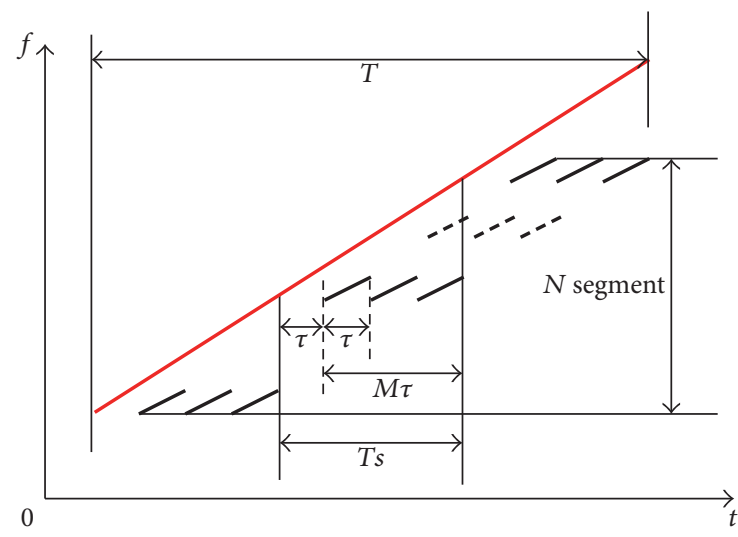

— Target

(a) Before dechirping processing

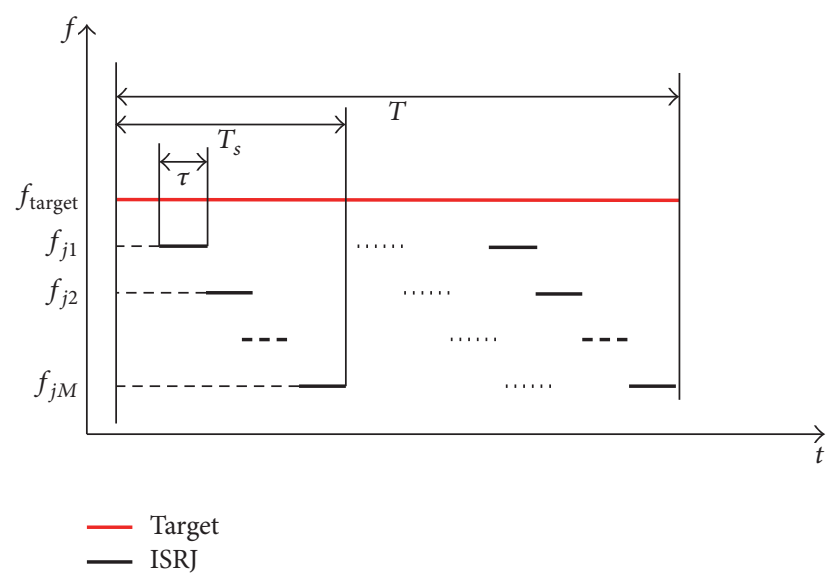

(b) After dechirping processing

FIGURE 2: TF distributions of the target echo signal and the ISRJ signal.

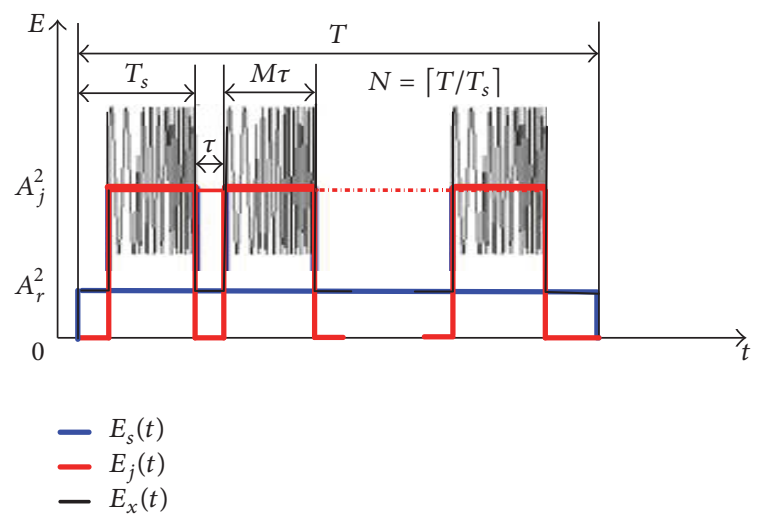

FIgURE 3: Energy functions.

3.2. How to Determine the Threshold Value. Not considering the noise or when the noise is small enough and can be negligible, we can get that $\left(A_{j}-A_{r}\right)^{2} \leq E_{x}(t) \leq\left(A_{j}+A_{r}\right)^{2}$ according to (12). Then, when $\left(A_{j}-A_{r}\right)^{2}>A_{r}^{2}$, that is, $A_{j}>2 A_{r}$ (this is a necessary condition to form an effective jamming), we always can find a threshold value $\gamma$, satisfying $A_{r}^{2}<\gamma<\left(A_{j}-A_{r}\right)^{2}$. Then the target echo signal segments not disturbed by the ISRJ signal can be found by comparing $E_{x}(t)$ with $\gamma$ which can be set as

$$
\gamma=\operatorname{mean}\left(\operatorname{envminp}\left(E_{x}(t)\right)\right),
$$

where $\operatorname{envminp}\left(E_{x}(t)\right)$ represents the minimum envelope of the energy function and mean $(\cdot)$ denotes the expectation operation.

$$
\begin{aligned}
& \text { For } \\
& \begin{array}{l}
\operatorname{envminp}\left(E_{x}(t)\right)=\sum_{n=0}^{N-1}\left(A_{r}^{2} \operatorname{rect}\left(\frac{t-\tau / 2-n T_{s}}{\tau}\right)\right. \\
\left.+\left(A_{j}-A_{r}\right)^{2} \operatorname{rect}\left(\frac{t-(1+M / 2) \tau-n T_{s}}{M \tau}\right)\right)
\end{array}
\end{aligned}
$$

we have

$$
\begin{aligned}
\gamma & =\operatorname{mean}\left(\operatorname{envminp}\left(E_{x}(t)\right)\right) \\
& =\frac{A_{r}^{2} \cdot \tau+\left(A_{j}-A_{r}\right)^{2} \cdot\left(T_{s}-\tau\right)}{T_{s}} \\
& =\left(A_{j}-A_{r}\right)^{2}-\frac{\tau}{T_{s}} A_{j}\left(A_{j}-2 A_{r}\right) \\
& =A_{r}^{2}+\left(1-\frac{\tau}{T_{s}}\right)\left(A_{j}^{2}-2 A_{j} A_{r}\right)
\end{aligned}
$$


and generally $0<\tau / T_{s} \leq 1 / 2<1$, so $\gamma=$ mean(envminp $\left.\left(p_{x}(t)\right)\right)$ satisfies $A_{r}^{2}<\gamma<\left(A_{j}-A_{r}\right)^{2}$.

When the noise is not small enough and cannot be negligible, the envelope of the energy function is not constant any more. There will be large error using the above methods of determining the threshold value and extracting the signal segments not disturbed by the ISRJ signal. Then an improved method is proposed as follows:

(1) Calculating the maximum and the minimum envelopes of the energy function, $\operatorname{envmaxp}\left(E_{x}(t)\right)$ and $\operatorname{envminp}\left(E_{x}(t)\right)$, respectively

(2) Calculating the mean envelope of the maximum and the minimum envelopes:

$\operatorname{envmoyp}\left(E_{x}(t)\right)$

$$
=\frac{\left(\operatorname{envmaxp}\left(E_{x}(t)\right)+\operatorname{envminp}\left(E_{x}(t)\right)\right)}{2}
$$

(3) Comparing $\operatorname{envmoyp}\left(E_{x}(t)\right)$ with $\gamma$ : when $\operatorname{envmoyp}\left(E_{x}\left(t_{0}\right)\right)<\gamma, p\left(t_{0}\right)=1$; otherwise, $p\left(t_{0}\right)=$ 0 . According to (13), we can get the signal segments not disturbed by the ISRJ signal

This method can maximally avoid the influence of noise on extracting the signal segments not disturbed by the ISRJ signal.

3.3. The Improved Method of Designing the Band-Pass Filtering Function $H(f)$. Theoretically, $p(t)$ should be a rectangular envelope pulse train with pulse duration $\tau$ and pulse repeat sampling interval $T_{s}$, which can be defined by

$$
h(t)=x(t) \cdot p(t)=s_{t \_\mathrm{de}}(t) \cdot p(t),
$$

where $p(t)=\operatorname{rect}((t-\tau / 2) / \tau) * \sum_{n=0}^{N-1} \delta\left(t-n T_{s}\right), *$ denotes convolution operator. are

The frequency spectrums of $p(t)$ and $s_{t \_ \text {de }}(t)$, respectively,

$$
\begin{aligned}
P(f) & =\sum_{n=0}^{N-1} \tau f_{s} \sin c\left(n f_{s} \tau\right) e^{-j \pi n f_{s} \tau} \delta\left(f-n f_{s}\right) \\
& =\sum_{n=0}^{N-1} a_{n} \delta\left(f-n f_{s}\right), \\
S_{t_{-} \text {de }}(f) & =A_{r} T \sin c\left[\left(f-f_{t_{-} \text {de }}\right) T\right] e^{j\left[\varphi_{0}-\pi\left(f-f_{t_{-} \mathrm{de}}\right) T\right]},
\end{aligned}
$$

where $\varphi_{0}=-2 \pi f_{c}\left(\tau_{t}-\tau_{\text {ref }}\right)+\pi k\left(\tau_{t}^{2}-\tau_{\text {ref }}^{2}\right)$. Then the frequency spectrum of $h(t)$ is

$$
H(f)=\sum_{n=0}^{N-1} a_{n} S_{t \_\mathrm{de}}\left(f-n f_{s}\right),
$$

where $f_{s}=1 / T_{s}$.

From $H(f)$, we can clearly see that the frequency $f_{\max }$ corresponding to the maximum value of $|H(f)|$ is just $f_{t_{-} \text {de }}$. But there exist high sidelobes beside the mainlobe which may
TABLE 1: Simulation parameters.

\begin{tabular}{lc}
\hline Parameter & Value \\
\hline Peak power of radar $P_{t} / \mathrm{MW}$ & 1.5 \\
Radar antenna gain $G / \mathrm{dB}$ & 45 \\
Wavelength $\lambda / \mathrm{cm}$ & 5 \\
Noise temperature $T_{e} / \mathrm{K}$ & 290 \\
Noise figure $F / \mathrm{dB}$ & 3 \\
Radar loss $L / \mathrm{dB}$ & 6 \\
Bandwidth of radar LFM signal $B / \mathrm{MHz}$ & 10 \\
Radar pulse width $T / \mu \mathrm{s}$ & 128 \\
Radar IF sampling frequency $f_{s} / \mathrm{MHz}$ & 5 \\
Target RCS $\sigma / \mathrm{m}^{2}$ & 1 \\
Distance between target (jammer) and & \\
radar $R_{t} /$ km & 100 \\
(self-defense jamming) & \\
Transmit power of jammer $P_{j} / \mathrm{W}$ & 70 \\
Jammer sampling pulse width $\tau / \mu \mathrm{s}$ & 1 \\
Jammer repeat sampling interval $T_{s} / \mu \mathrm{s}$ & 2 and 10 \\
\hline
\end{tabular}

cause the result that there are still some strong false targets after filtering and the radar system still cannot detect the true target correctly.

In this paper, the method of windowing in the frequency domain is designed as follows to suppress the sidelobes of $H(f)$.

Step 1. Choose a window function $w(t)$, such as hamming window. By doing Fourier transform on $w(t)$, we get its frequency spectrum $W(f)$ whose center frequency is located in zero.

Step 2. Constructing a one-dimensional optimization function,

$$
g\left(f_{0}\right)=\sum_{f}\left|W\left(f-f_{0}\right) \cdot H(f)\right| .
$$

And find $f_{0 \max }$ that makes $g\left(f_{0}\right)$ get the maximum value.

$$
f_{0 \max }=\max _{f_{0}} g\left(f_{0}\right) .
$$

Then, the window function in frequency domain can be obtained as

$$
W_{a}(f)=W\left(f-f_{0 \max }\right) .
$$

Step 3. Multiplying $W_{a}(f)$ and $H(f)$ to get the filtering function with low sidelobes $H_{a}(f)$,

$$
H_{a}(f)=W_{a}(f) \cdot H(f) .
$$

\section{Simulations}

In this section, we analyze the performance of the proposed ECCM scheme to the ISRJ through some simulations. The simulation parameters employed are illustrated in Table 1. Here, the system delay of the jammer is too small to consider. 


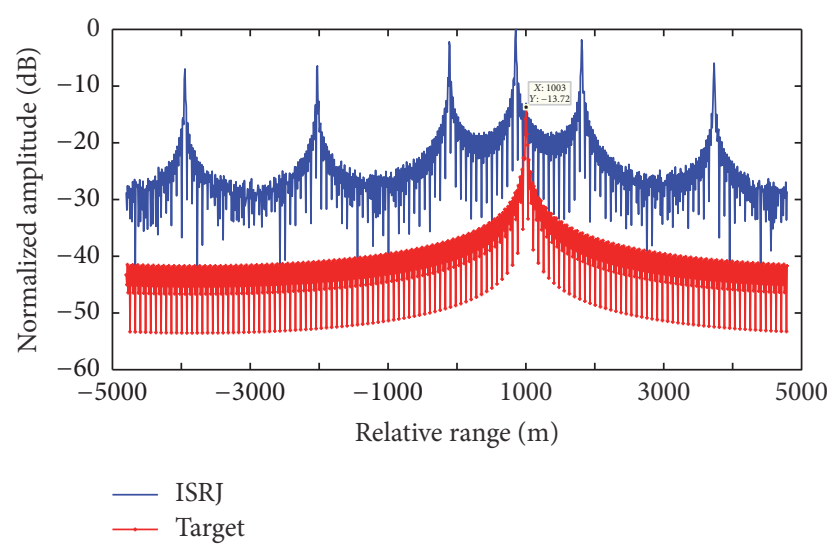

FIGURE 4: The compression result before $\operatorname{ECCM}\left(T_{s}=2 \mu \mathrm{s}\right)$.

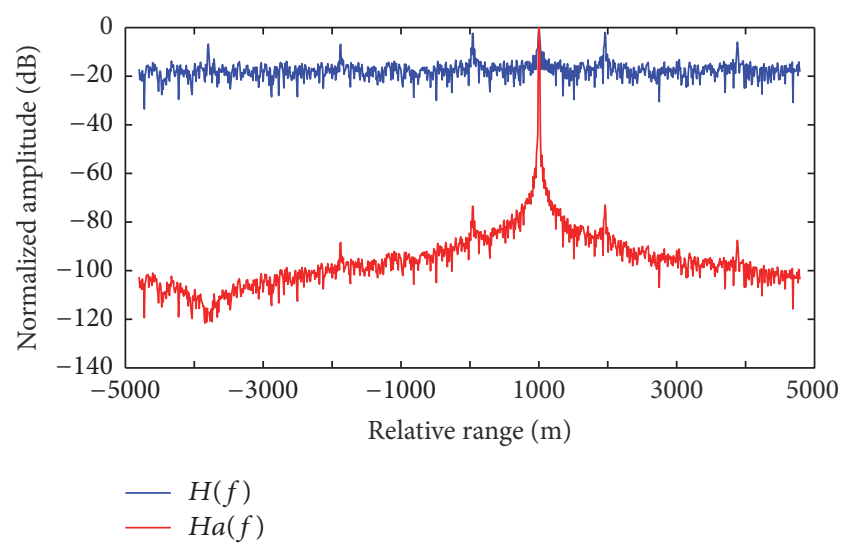

Figure 5: The band-pass filtering function $\left(T_{s}=2 \mu \mathrm{s}\right)$.

The influences of the jamming repeat sampling interval $T_{s}$ and the duty ratio $\tau / T_{s}$ on the jamming performance of ISRJ have been studied in [22]. The distance between the false targets and the amplitudes of the false targets are determined by the jamming repeat sampling interval $T_{s}$ and the duty ratio $\tau / T_{s}$, respectively. Higher $T_{s}$ produces false targets intensively distributed, and smaller $T_{s}$ generates false targets sparsely distributed. There are only three to five strong false targets while the amplitudes of the high-order false targets attenuate rapidly under the high duty ratio conditions. A large number of false targets with slowly attenuated amplitudes and more serious power loss are generated under the small duty ratio conditions. Assume that the jamming sampling duration $\tau=$ $1 \mu \mathrm{s}$ is invariable in the following simulations.

When $T_{s}=2 \mu \mathrm{s}$, that is, the duty ratio is $50 \%$, the theoretical analysis and the simulation result shown in Figure 4 reveal that there exist three strong lifelike false targets resulting in a confusion that the radar system cannot judge which one is the true target. The $x$-axis in the simulation figures is represented by the relative distance to reference point as a result of the proportional relationship with the single-frequency target echo signal after dechirping.

Figure 5 shows the magnitude-frequency responses of the filters before and after windowing in frequency domain. It can be seen from Figure 5 that the sidelobes of the

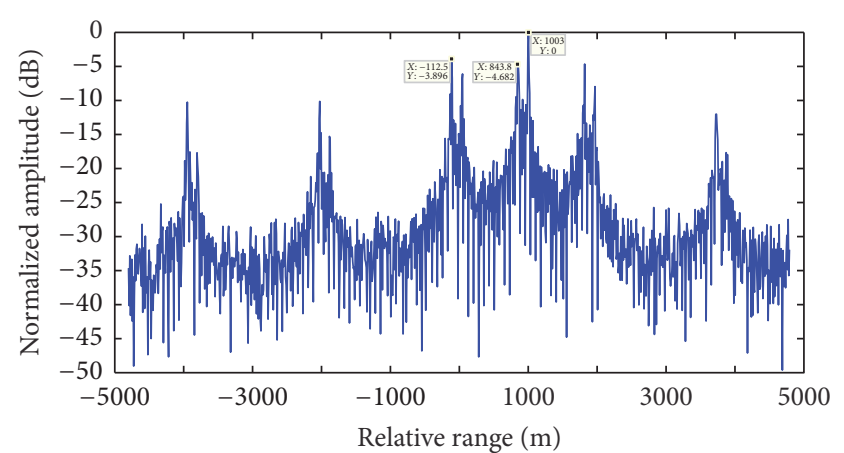

FIgURE 6: The jamming suppression result using $H(f)\left(T_{s}=2 \mu \mathrm{s}\right)$.

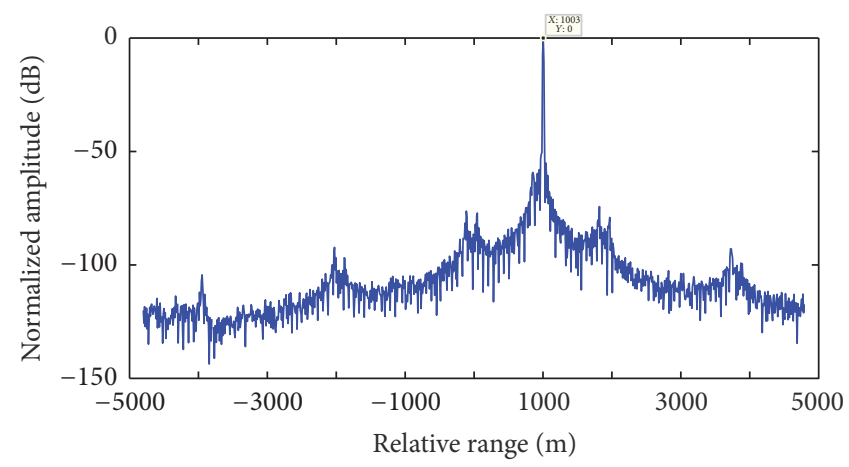

FIGURE 7: The jamming suppression result using $H_{a}(f)\left(T_{s}=2 \mu \mathrm{s}\right)$.

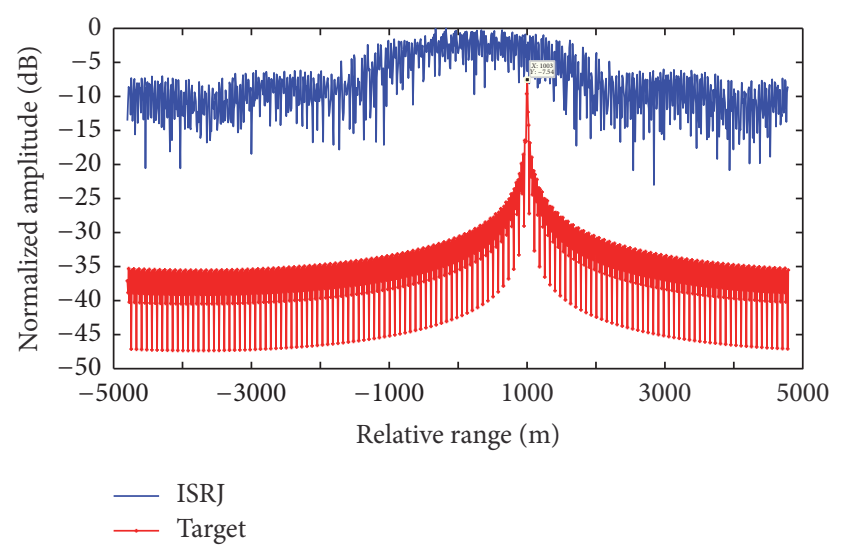

FIGURE 8: The compression result before $\operatorname{ECCM}\left(T_{s}=10 \mu s\right)$.

filtering function are suppressed by a wide margin through windowing in frequency domain.

The suppression result with the filter not windowed is shown in Figure 6. It can be seen that there still exist one strong false target for high sidelobes resulting in a confusion to the radar system. Figure 7 shows the suppression result with the windowed filter. From Figure 7, we can see that all false targets are removed for the extremely low sidelobes of the windowed filter.

When $T_{s}=10 \mu \mathrm{s}$, that is, the duty ratio is $10 \%$, the theoretical analysis and the simulation result shown in Figure 8 illustrate that the intensively distributed false targets are generated resulting in a confusion to the radar system. 


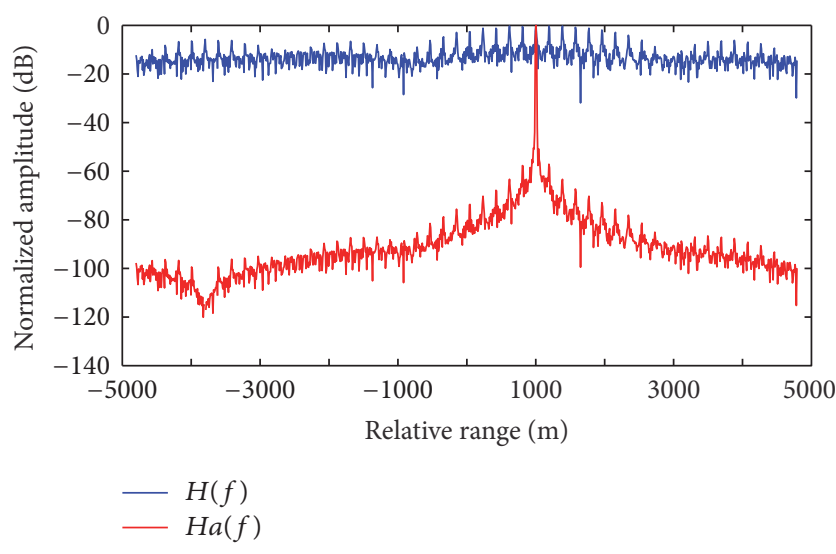

FIGURE 9: The band-pass filtering function $\left(T_{s}=10 \mu s\right)$.

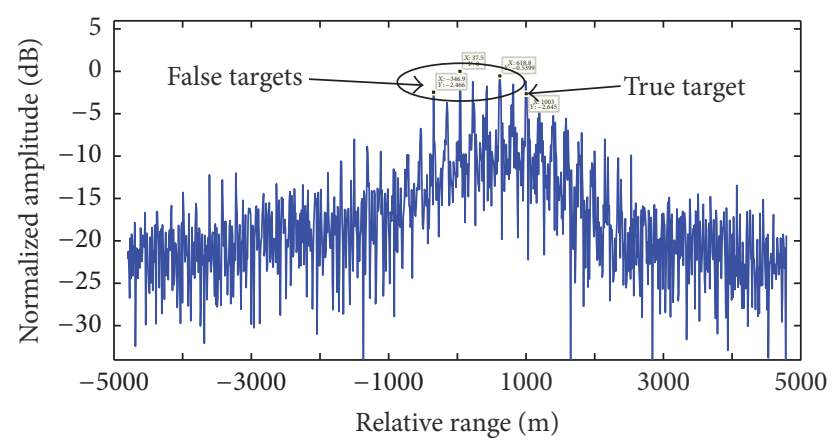

FIGURE 10: The jamming suppression result using $H(f)\left(T_{s}=10 \mu \mathrm{s}\right)$.

Similarly, Figure 9 shows the magnitude-frequency response of the filters before and after windowing in frequency domain and has similar interpretation to Figure 5.

The suppression result with the filtering function not windowed is shown in Figure 10. It can be seen that there are still many false targets left surrounding the true target for high sidelobes. Figure 11 shows the suppression result with the windowed filtering function. From Figure 11, we can see that the suppression efficiency is obviously good for the extremely low sidelobes of the windowed filtering function.

Signal-to-noise ratio (SNR) and jamming-to-signal ratio (JSR) will affect the validity of the proposed ECCM method. SNR and JSR are defined as

$$
\begin{gathered}
\mathrm{SNR}=10 \log _{10}\left(\frac{A_{r}^{2}}{\sigma^{2}}\right), \\
\mathrm{JSR}=10 \log _{10}\left(\frac{A_{j}^{2}}{A_{r}^{2}}\right),
\end{gathered}
$$

where $\sigma^{2}$ represents the power of noise. Then under different JSR, assume that the peak value of the compression result after executing the ECCM scheme proposed above is the target and make the Monte-Carlo simulation 1000 times in every SNR. When the noise power is increasing gradually, Figure 12 shows the detection probability of the true target.

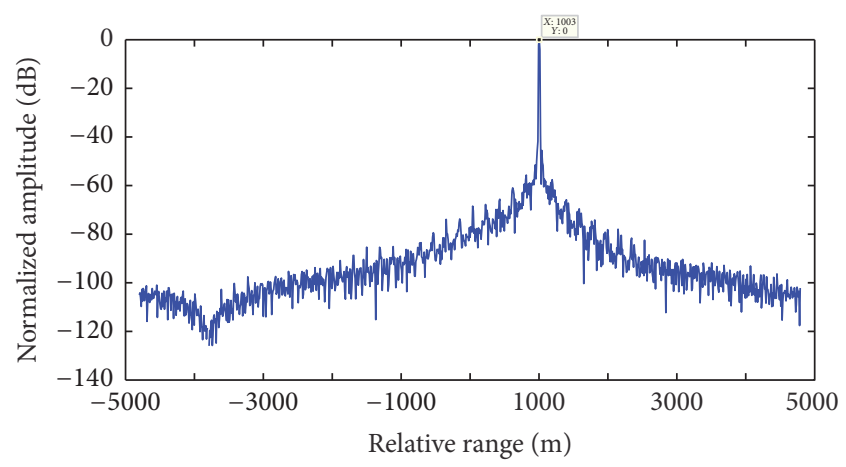

FIGURE 11: The jamming suppression result using $H_{a}(f)\left(T_{s}=\right.$ $10 \mu \mathrm{s})$.

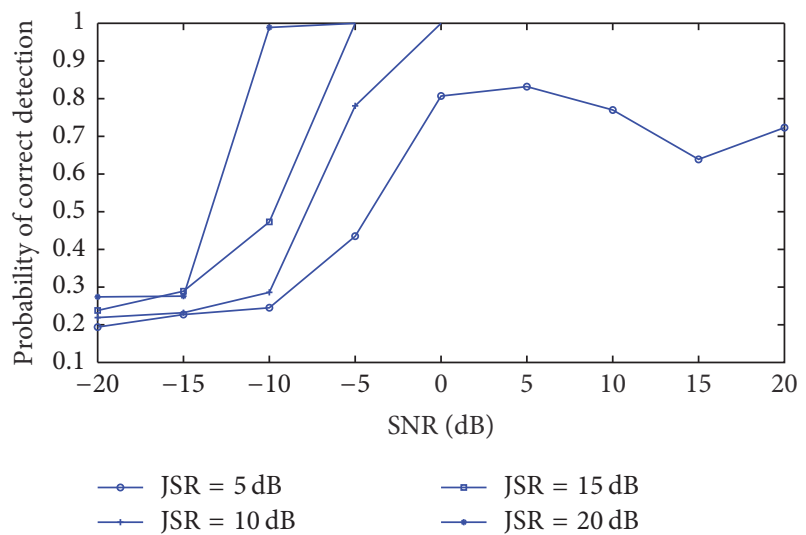

FIGURE 12: Detection probability of the true target with different SNR under different JSR.

From Figure 12, it can be seen that the larger JSR, the larger detection probability of the true target. Under the condition of JSR $=10 \mathrm{~dB}$, JSR $=15 \mathrm{~dB}$ and JSR $=20 \mathrm{~dB}$, the true target can be detected with almost $100 \%$ probability when SNR > $0 \mathrm{~dB}$, SNR > $-5 \mathrm{~dB}$ and SNR $>-10 \mathrm{~dB}$, respectively. The larger JSR is beneficial to extract the signal segments not disturbed by the ISRJ signal and construct the band-pass filter exactly.

\section{Conclusions}

Based on the discontinuity characteristics of the ISRJ signal in TF domain and the great differences in time-energy domain, a simple and effective ECCM scheme against the ISRJ is proposed in this paper. Firstly, a simple energy function is defined and used to extract the target echo signal segments without jamming. It is not necessary to perform the complicated time-frequency decomposition. Then, the band-pass filtering function with low sidelobes is obtained by doing the Fourier transformation directly on the extracted signal and windowing in frequency domain. The simulation results show that the proposed method is effective not only in multiple lifelike false targets, but also in intensively distributed false targets generated through setting different jamming repeat sampling intervals. Moreover, this method is effective even 
with low SNR under certain JSR, and we can acquire the detection probability of almost $100 \%$ to the true target.

\section{Conflicts of Interest}

The authors declare that there are no conflicts of interest regarding the publication of this paper.

\section{Acknowledgments}

This work was granted partially by National Natural Science Foundation of China (61501500).

\section{References}

[1] X. Wang, J. Liu, W. Zhang, Q. Fu, Z. Liu, and X. Xie, "Mathematic principles of interrupted-sampling repeater jamming (ISRJ)," Science in China, Series F: Information Sciences, vol. 50, no. 1, pp. 113-123, 2007.

[2] M. J. Sparrow and J. Cakilo, "ECM techniques to counter pulse compression radar," United States Patent, 7081846, 2006.

[3] M. Soumekh, "SAR-ECCM using phase-perturbed LFM chirp signals and DRFM repeat jammer penalization," IEEE Transactions on Aerospace and Electronic Systems, vol. 42, no. 1, pp. 191205, 2006.

[4] X.-Z. Feng and X.-J. Xu, "Study of countermeasures to deceptive jamming using random linear modulation frequency ratio SAR," Systems Engineering and Electronics, vol. 31, no. 1, pp. 6973, 2009 (Chinese).

[5] J. Akhtar, "An ECCM signaling approach for deep fading of jamming reflectors," in Proceedings of the IET International Conference on Radar Systems, pp. 1-5, Edinburgh, UK, October 2007.

[6] J. Akhtar, "Orthogonal block coded ECCM schemes against repeat radar jammers," IEEE Transactions on Aerospace and Electronic Systems, vol. 45, no. 3, pp. 1218-1226, 2009.

[7] J. Schuerger and D. Garmatyuk, "Performance of random OFDM radar signals in deception jamming scenarios," in Proceedings of the IEEE Radar Conference, pp. 1-6, May 2009.

[8] J. D. Zhang, X. H. Zhu, and K. R. Wang, "A waveform diversity technique for countering RGPO," in Proceedings of the IET International Radar Conference, pp. 1-4, Guilin, China, April 2009.

[9] Z. Liu, X.-S. Wang, J.-C. Liu, G.-Y. Wang, and S.-P. Xiao, "Jamming technique of interrupted-sampling and periodic repeater based on digital radio frequency memory," Acta Armamentarii, vol. 29, no. 4, pp. 405-410, 2008 (Chinese).

[10] Z. Zhou, H. Tang, Y. S. Zhang, and C. Y. Wang, "A study on smart noise jamming based on time domain sampling," Modern Radar, vol. 32, no. 5, pp. 53-55, 2010 (Chinese).

[11] J.-C. Liu, X.-S. Wang, Z. Liu, J.-H. Yang, and G.-Y. Wang, "Preceded false target groups jamming against LFM pulse compression radars," Journal of Electronics and Information Technology, vol. 30, no. 6, pp. 1350-1353, 2008 (Chinese).

[12] Y.-R. Zhang, Y.-J. Li, M.-L. Li, M.-G. Gao, and X.-J. Fu, "Suppress jamming technique of multiple false targets on interrupted-sampling and non-uniform periodic repeater," Acta Electronica Sinica, vol. 44, no. 1, pp. 46-53, 2016 (Chinese).

[13] X. S. Wang, S. P. Xiao, D. J. Feng et al., Modeling and Simulation of Modern Radar and Electronic Warfare Systems, Publishing House of Electronic Industry, Beijing, China, 2010 (Chinese).
[14] D. J. Feng, L. T. Xu, and X. S. Wang, "Phase signature of active decoy and its application in angular deception jamming using interrupted-sampling repeater," Journal of National University of Defense Technology, vol. 36, no. 3, pp. 135-140, 2014 (Chinese).

[15] D. Feng, Y. Yang, and L. Xu, "Impact analysis of CFAR detection for active decoy using interrupted-sampling repeater," Journal of National University of Defense Technology, vol. 38, no. 1, pp. 63-68, 2016.

[16] D. J. Feng, H. M. Tao, Y. Yang, and Z. Liu, "Jamming dechirping radar using interrupted-sampling repeater," Science China: Information Sciences, vol. 54, no. 10, pp. 2138-2146, 2011.

[17] L. Xu, D. Feng, W. Zhang, and X. Wang, "Group targets generation against ISAR based on intermittent-sampling repeater jamming (ISRJ)," Journal of National University of Defense Technology, vol. 35, no. 5, pp. 140-145, 2013 (Chinese).

[18] X.-Y. Pan, W. Wang, D.-J. Feng, Q.-X. Fu, and G.-Y. Wang, "Jamming dechirping ISAR based on intermittent sampling repeater," Journal of Astronautics, vol. 34, no. 9, pp. 1274-1280, 2013 (Chinese).

[19] Y. Wang, C. Y. Shu, S. J. Zhang, P. Huang, and J. Ji, “Array ISAR of precessional cone target generated by intermittent sampling repeater jamming in fast and slow time," Journal of Electronics and Information Technology, vol. 38, no. 2, pp. 450-454, 2016 (Chinese).

[20] Y. Jiang, M. H. He, H. B. Liu, and C. L. Yu, "Recognition of interrupted-sampling repeater jamming based on resemblance coefficient," Modern Radar, vol. 38, no. 5, pp. 72-76, 2016 (Chinese).

[21] S. X. Gong, X. Z. Wei, and X. Li, "ECCM scheme against interrupted sampling repeater jammer based on time-frequency analysis," Journal of Systems Engineering and Electronics, vol. 25, no. 6, pp. 996-1003, 2014.

[22] N. E. Huang, Z. Shen, S. R. Long et al., “The empirical mode decomposition and the Hilbert spectrum for nonlinear and non-stationary time series analysis," Proceedings of the Royal Society A: Mathematical, Physical and Engineering Sciences, vol. 454, no. 1971, pp. 903-995, 1998. 


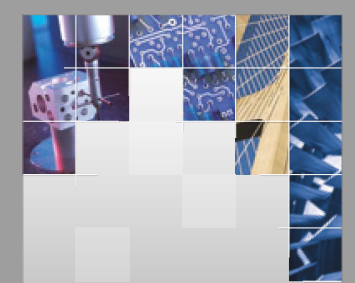

\section{Enfincering}
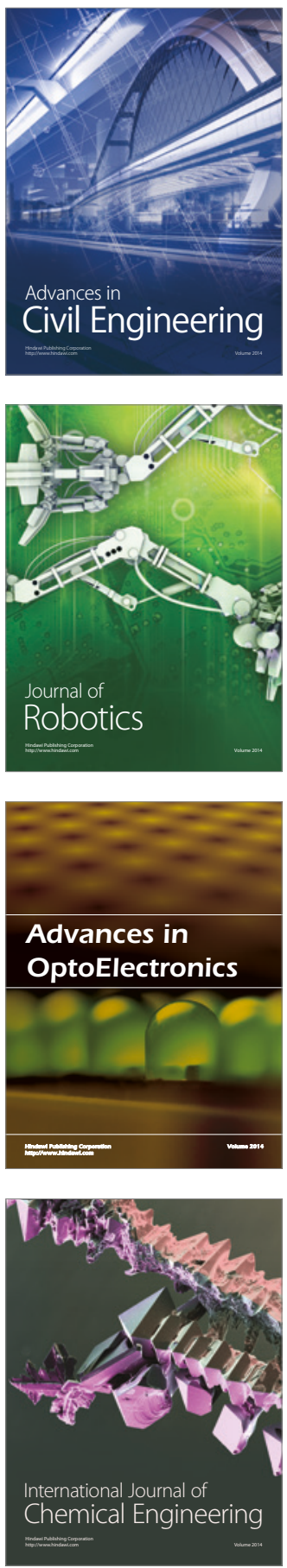

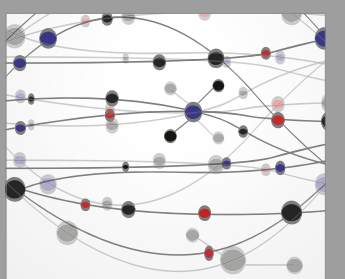

The Scientific World Journal

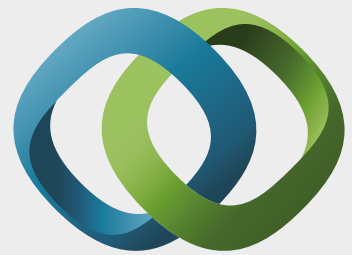

\section{Hindawi}

Submit your manuscripts at

https://www.hindawi.com
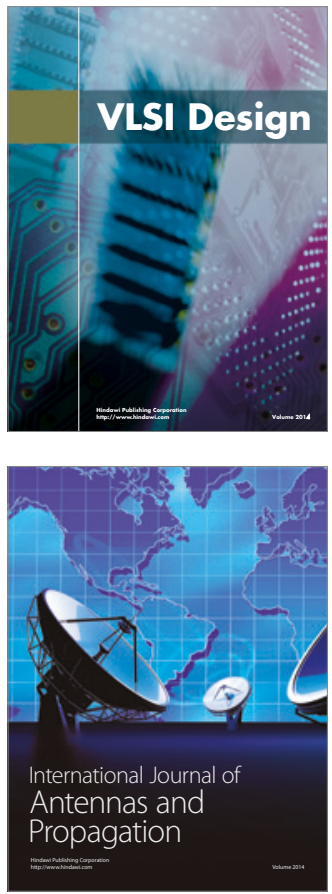

\section{Rotating}

Machinery
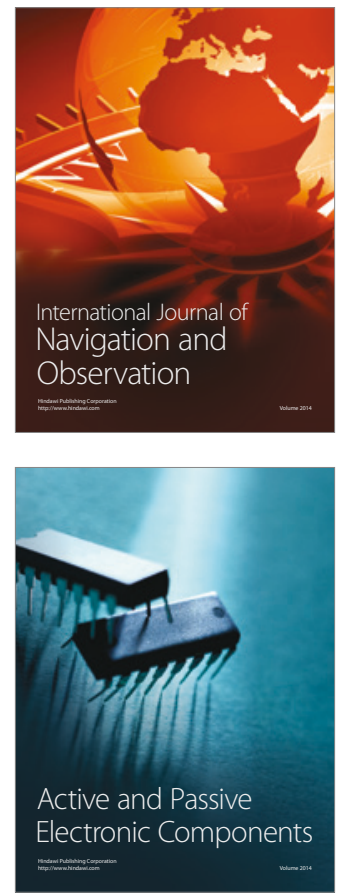
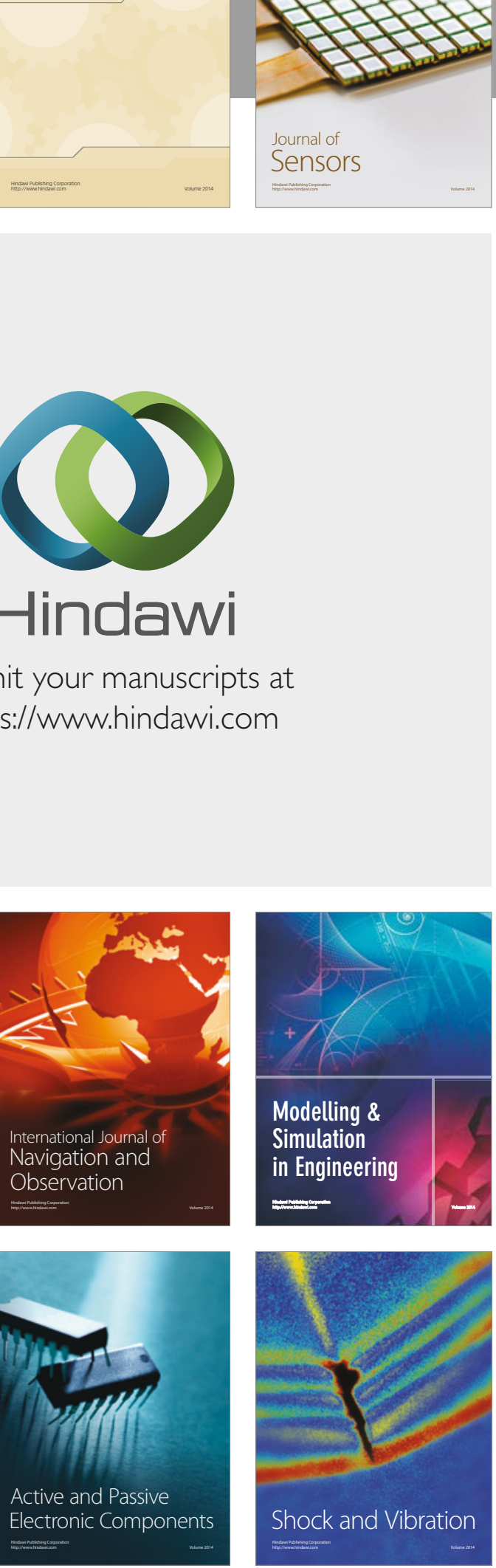
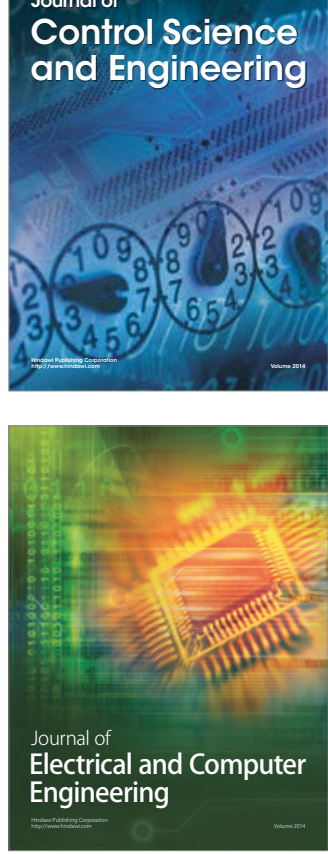

Distributed

Journal of

Control Science

and Engineering
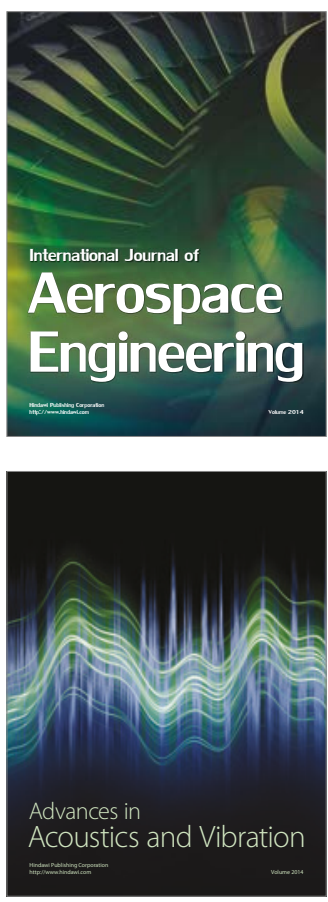

Sensor Networks 